\section{Estudo das internações \\ hospitalares por pneumoconioses no Brasil, 1993-2003}

\author{
Hospital admissions due to \\ pneumoconiosis in Brazil, 1993-2003
}

\section{Resumo}

Possivelmente, a incidência de pneumoconiose deve ser elevada entre os expostos, mas não há informações epidemiológicas referentes a séries históricas sobre as internações hospitalares nas diversas regiões do país. Objetivo: Estudar a freqüência e distribuição dessa doença no Brasil e suas unidades federadas, através de levantamento das internações hospitalares por pneumoconiose no período entre 1993 e 2003, e discutir as possibilidades de estas internações estarem relacionadas ao processo de trabalho de cada região. Material e Métodos: Informações de morbidade por pneumoconiose foram recolhidas, descritas e analisadas a partir do Sistema de Informação das Internações Hospitalares do SUS e do IBGE, para população acima de 15 anos, distribuídas por Estados e no Brasil no período de 1993 a 2003. Foram utilizadas listas da CID-9 (1993-1997) e da CID-10 (1998-2003). Resultados: Observou-se uma predominância dos Estados do Sul e Sudeste, demonstrando uma correlação direta com o processo de trabalho e o acesso dos trabalhadores aos serviços de saúde. Discussão: Os dados permitiram identificar uma diferença entre os resultados anteriores e, após 1998, com a mudança da CID 9 para a CID 10 , indicando uma relação entre as modificações de critérios diagnósticos e na forma de coleta dos dados primários pelo SUS. Conclusão: A distribuição das internações é heterogênea e provavelmente reflete o vértice de uma pirâmide invertida do total de casos que deve ocorrer, de fato, entre os trabalhadores brasileiros. Seu enfrentamento possibilita a aproximação da real situação epidemiológica, contribuindo para a orientação de medidas profiláticas de redução dos riscos e preparo do sistema assistencial.

Palavras-chave: Pneumoconiose. Silicose. Asbestose. Doença respiratória ocupacional.

Financiamento: CNPq / Bolsa PIBIC

Correspondência: Hermano Albuquerque de Castro. Rua Leopoldo Bulhões, 1480 - Manguinhos, Rio de Janeiro, RJ CEP 21041-210. E-mail: castro@ensp.fiocruz.br 
Abstract

The incidence of pneumoconiosis is probably high among exposed workers, but there is no epidemiological information such as a historical series of hospitalizations in the several areas of the country. Objective: to study the frequency and distribution of this disease, in Brazil and its states, through the survey of hospital admissions due to pneumoconiosis in the period between 1993 and 2003, and to discuss the possibilities that these admissions were related to the work processes of each region. Methods: pneumoconiosis morbidity information was collected from the Hospital Admission Information System of SUS and IBGE (Brazilian Institute of Geography and Statistics), described and analyzed, for the population over 15 years of age in the period between 1993 and 2003, distributed per state and Brazil in the period between 1993 and 2003. ICD-9 (19931997) and ICD-10 (1998-2003) were used. Results: a predominance of the states of the South and Southeast was observed, showing a correlation with work processes and access of workers to healthcare. Discussion: The data permitted identifying a difference between the data before and after 1998, which corresponds to the change from ICD-9 to ICD-10, thus indicating a correlation between changes in diagnostic criteria and the form of collecting primary data by the SUS. Conclusion: The distribution of hospital admissions is heterogeneous and this possibly reflects the vertex of an inverted pyramid of the total of cases that should in fact occur among Brazilian workers. Facing the situation makes it possible to approach the actual epidemiological status, and thus contribute to the orientation of prophylactic and risk-reduction measures and preparation of the health system.

Keywords: Pneumoconiosis. Silicosis. Asbestosis. Occupational lung disease.

\section{Introdução}

As pneumoconioses representam um grupo de doenças causadas pela inalação e acúmulo de poeira nos pulmões, que acarretam uma reação granulomatosa pulmonar. O processo fisiopatológico iniciase quando a poeira inalada atinge o parênquima pulmonar, atraindo células fagocitárias e de defesa para o local, ocasionando a liberação de substâncias quimiotáxicas e também fibrogênicas, dando início à lesão silicótica, constituída por camadas de tecido hialino, que tem de permeio quantidade razoável de poeira ${ }^{1}$.

Cada pneumoconiose recebe um nome particular, de acordo com a poeira inalada, representada por talcos, sílicas, asbesto, ferro, estanho e tantas outras poeiras minerais. Sendo assim, a doença causada por sílica chama-se silicose, asbesto asbestose, ferro - siderose, estanho estanhose, talco - talcose, e cada uma delas recebe um código na Classificação Internacional de Doenças (CID).

No Brasil, em função da multiplicidade de atividades extrativistas e industriais, existe um número elevado de trabalhadores expostos às poeiras minerais capazes de produzir essas doenças, constituindo um problema de saúde pública.

A principal pneumoconiose no país, do ponto de vista epidemiológico e de Saúde Pública, é a silicose, causada pela exposição à poeira de sílica livre ou dióxido de silício (SIO2) em sua forma cristalina ${ }^{2}$.

A asbestose é outra pneumoconiose caracterizada por produzir agravos importantes. Tem origem mineral, é incombustível, resistente à geração de calor e fricção, à corrosão e à maior parte dos ácidos e outras propriedades que garantem sua utilização em mais de três mil (3.000) produtos $^{3}$.

No país, por suas características e pelas imensas reservas naturais, o amianto tem sido usado em larga escala há muitas décadas. Estima-se que o número de pessoas direta e ocupacionalmente expostas seja de quinhentas mil (500.000), das quais 
cerca de duzentas mil (200.000) são trabalhadores da indústria de extração e transformação - mineração, cimento-amianto, materiais de fricção e outros. Entretanto, segundo estimativa do Sindicato Nacional da Indústria de Autopeças, SINDIPEÇAS, há cerca de outros trezentos mil (300.000) trabalhadores envolvidos em manutenção e reparos de sistemas de freio no país, e uma parcela ainda maior, desconhecida, de trabalhadores informais, principalmente envolvidos na indústria da construção civil, em atividades como instalação de coberturas, caixas d'água, reformas, demolições, instalações hidráulicas etc., que estão completamente à margem de qualquer proteção social e das incipientes políticas públicas de saúde do trabalhador ${ }^{2}$.

Estima-se um grande número de trabalhadores sob o risco de desenvolver tais doenças, principalmente considerando o elevado contingente de indústrias que utilizam minerais com potencial fibrogênico em seu processo produtivo. Alia-se a este fator a possibilidade de associação com outras doenças, como é o caso da tuberculose e do câncer pulmonar.

Assim, os objetivos são estudar a freqüência e distribuição dessa doença no Brasil e suas unidades federadas, pelo levantamento das internações hospitalares por pneumoconiose no período entre 1993 e 2003, e discutir as possibilidades de estas internações estarem relacionadas ao processo de trabalho de cada região.

\section{Material e Métodos}

Trata-se de um estudo epidemiológico descritivo. As informações das internações hospitalares por pneumoconiose foram obtidas através do Sistema de Internações Hospitalares (SIH) do SUS, no período de 1993 a 2003. Os dados das internações distribuídos para os Estados e Brasil foram divididos pela população de maiores de quinze anos para cálculo dos coeficientes nacional e estadual, os quais se buscou apontar as atividades com exposição.

A codificação das causas das inter- nações hospitalares foi realizada segundo as normas da Nona Revisão da Classificação Internacional de Doenças (CID 9), para o período 1993-1997 e, pela Décima Revisão da Classificação Internacional de Doenças, Agravos e Problemas Relacionados à Saúde (CID-10), após 1998. No período de vigência da Nona Revisão, a utilização dos códigos com três dígitos foi a forma mais adequada de seleção das internações hospitalares por pneumoconioses, incluindo-se os códigos 500; 501; 502; 503 e 505, do capítulo 08 da (CID 9). Optou-se pela exclusão do código 504 (Pneumopatias devido à inalação de outras poeiras), pois engloba outras patologias além das pneumoconioses.

No período de vigência da (CID-10) (1996-2003) foram incluídos os códigos J60, J61, J62.8, J63, J63.1, J63.2, J63.3, J63.4 , J63.8, J64 e J65, do capítulo 10 daquela Revisão. O código J62.8 (Pneumoconiose devido a outras poeiras que contém sílica) substituiu o código J62, excluindo os dados referentes a Pneumoconiose devido ao pó de talco. Essa substituição foi proposta para possibilitar a correlação com os dados do código 502 (Pneumoconiose devido a outras sílicas e silicatos).

As internações foram agrupadas por Estados, e os coeficientes calculados por 1.000.000 habitantes/ano. O grupo etário de menores de quinze anos não foi incluído no denominador para o cálculo dos coeficientes por ser pouco provável a presença de pneumoconiose, devido ao longo período de latência no desenvolvimento da doença. Foram calculados também coeficientes de internação hospitalar de acordo com o sexo.

Os dados de população, para cálculo de coeficientes, foram obtidos dos Censos Gerais dos anos de 1980, 1991 e 2000, realizados pela Fundação IBGE, e das estimativas feitas para os anos intercensitários.

O uso de base geográfica para a distribuição das internações hospitalares permitiu a verificação da existência de concordância entre aquelas e a presença de processos de trabalho envolvendo a expo- 
sição às poeiras potencialmente causadoras das pneumoconioses.

A análise estatística e a elaboração de gráficos e mapas foram realizadas utilizando-se os softwares Microsoft Excel 2000 e Tabwin 3.4.

O levantamento de dados foi aprovado pelo Comitê de Ética em Pesquisa da Escola Nacional de Saúde Pública - ENSP/ FIOCRUZ, e não há nenhum interesse financeiro ou outro no assunto-questão do trabalho que possa ser considerado um conflito de interesses.

\section{Resultados}

Para retratar a distribuição das internações por pneumoconiose no país foi elaborado o Mapa 1, que mostra a soma das freqüências ocorridas no período entre 1993 e 2003, para o Brasil e suas unidades federadas. Observa-se maior concentração na Região Sudeste (São Paulo, Minas Gerais e Rio de Janeiro), totalizando 2.351 casos e, nos Estados do Sul, Santa Catarina e Rio Grande do Sul, com 1.578 casos.

Na Tabela 1 estão demonstrados os coeficientes de internações hospitalares para o Brasil e seus Estados que apresentaram maior freqüência para cada ano estudado. O critério de seleção foi baseado nas freqüências de pneumoconioses com valores acima de zero em mais da metade dos anos analisados.

Das onze pneumoconioses registradas, destacam-se cinco: Pneumoconiose dos Trabalhadores Expostos ao Carvão (códigos 500 e J60), Pneumoconiose devido a outras sílicas e silicatos (Silicose) (502 e J62.8), Pneumoconiose derivada de outras poeiras inorgânicas (503 e J63.8), Pneumoconiose não especificada (505 e J64) e

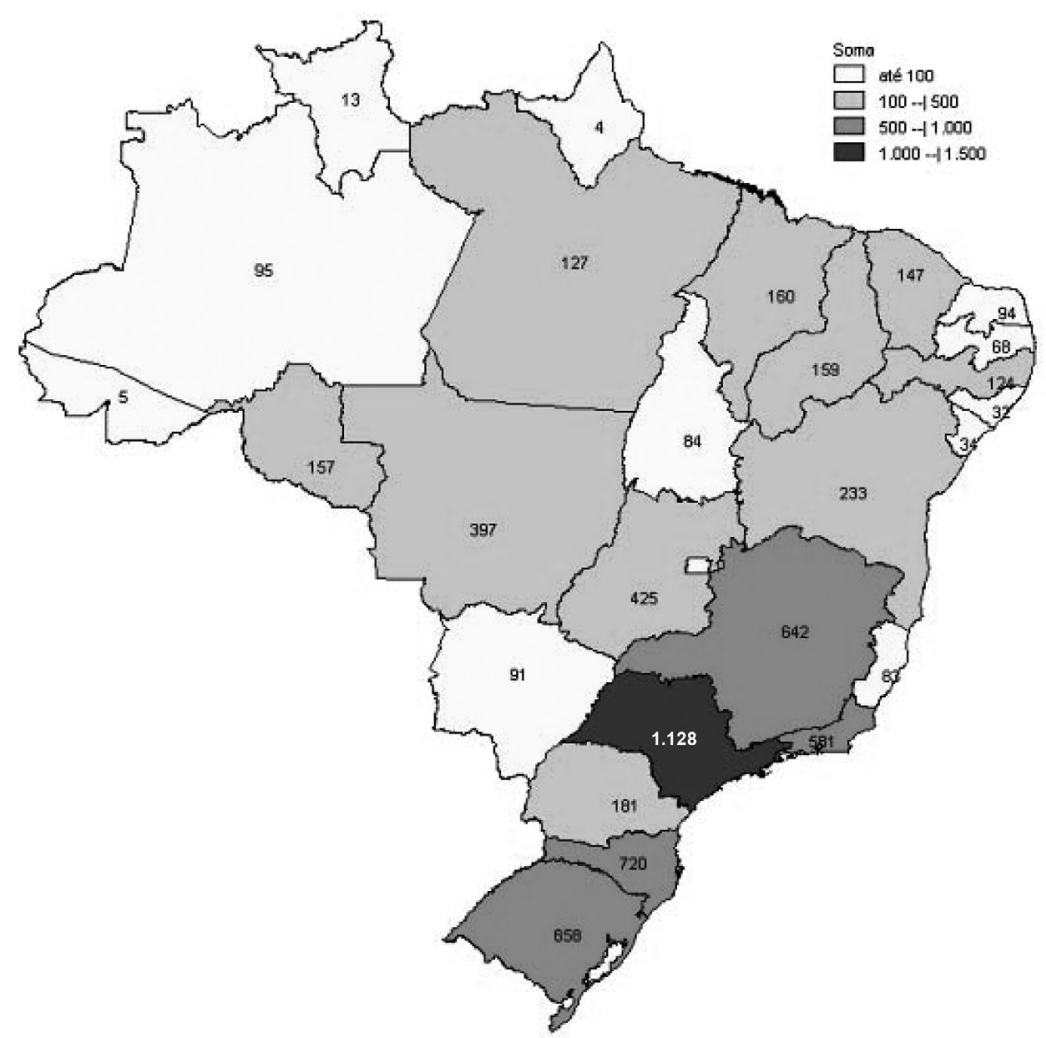

Mapa 1 - Número total das internações hospitalares por pneumoconioses no Brasil e nos estados, no período entre 1993 e 2003.

Map 1 - Total number of hospital admissions due to pneumoconiosis in Brazil, for the period between 1993 and 2003. 
Tabela 1 - Coeficientes das internações hospitalares por pneumoconioses, por milhão de habitantes/ano, Brasil e estados com maior freqüência, período entre 1993 e 2003.

Table 1 - Rates of hospital admissions due to pneumoconiosis per 1,000,000 inhabitants/year, Brazil and States with highest frequency, 1993 to 2003.

\begin{tabular}{|c|c|c|c|c|c|c|c|c|c|c|c|c|}
\hline CID & UF/Ano & 1993 & 1994 & 1995 & 1996 & 1997 & 1998 & 1999 & 2000 & 2001 & 2002 & 2003 \\
\hline \multirow[t]{6}{*}{500 e J60 } & Santa Catarina & 25,46 & 18,5 & 14,52 & 16,22 & 10,44 & 1,43 & 3,95 & 2,6 & 2,55 & 2,01 & 3,97 \\
\hline & Rio Grande do Sul & 9 & 10,71 & 11,94 & 12,23 & 8,38 & 0 & 0 & 0 & 0,13 & 0,78 & 1,8 \\
\hline & Goiás & 7,68 & 7,2 & 10,27 & 9,98 & 7,21 & 2,45 & 1,5 & 2,83 & 0,28 & 1,63 & 0 \\
\hline & Piauí & 10,84 & 3,15 & 7,46 & 7,09 & 5,27 & 0 & 0 & 0 & 0 & 0 & 0,51 \\
\hline & Tocantins & 7,06 & 6,92 & 11,91 & 13,77 & 1,48 & 0 & 0 & 0 & 0 & 0 & 0 \\
\hline & BRASIL & 2,71 & 3,22 & 4,02 & 2,72 & 2,09 & 0,23 & 0,21 & 0,22 & 0,21 & 0,26 & 0,31 \\
\hline \multirow[t]{6}{*}{502 e J62.8 } & Rio de Janeiro & 1,18 & 0,21 & 0,32 & 0,71 & 1,4 & 1,09 & 1,27 & 0,37 & 0,64 & 0,36 & 0,36 \\
\hline & Pernambuco & 2,53 & 0,09 & 1,65 & 0,78 & 1,88 & 0,84 & 0,33 & 0,16 & 0,08 & 0,08 & 0,75 \\
\hline & Rio Grande do Norte & 0,62 & 0,04 & 0,64 & 0,69 & 0,24 & 0,28 & 0,19 & 0,22 & 0,4 & 0,18 & 0,25 \\
\hline & Sergipe & 1,83 & 0 & 2,12 & 1,29 & 0,31 & 0,61 & 0,6 & 0,85 & 1,11 & 2,45 & 1,07 \\
\hline & Minas Gerais & 0 & 0 & 0 & 0 & 0 & 0 & 0 & 0,18 & 0 & 0 & 0 \\
\hline & BRASIL & 0,72 & 0,8 & 0,6 & 0,52 & 0,51 & 0,47 & 0,35 & 0,28 & 0,38 & 0,4 & 0,51 \\
\hline \multirow[t]{6}{*}{503 e J63.8 } & Mato Grosso & 17,06 & 2,14 & 11,79 & 6,11 & 7,96 & 1,3 & 0,64 & 0 & 0 & 0 & 0 \\
\hline & Minas Gerais & 0,94 & 0,09 & 0,37 & 0,69 & 0,94 & 0,08 & 0,17 & 0,16 & 0,08 & 0,08 & 0,08 \\
\hline & São Paulo & 1,5 & 0 & 1,2 & 1,06 & 1,28 & 0,91 & 0,93 & 0,55 & 0,51 & 0,18 & 0,07 \\
\hline & Rio Grande do Sul & 0,15 & 0 & 0 & 0,29 & 0,14 & 0,84 & 0,97 & 0,27 & 0,39 & 0,13 & 0 \\
\hline & Goias & 4,02 & 0,36 & 1,06 & 0 & 0,31 & 0,31 & 0,3 & 0 & 0 & 0,27 & 0,53 \\
\hline & BRASIL & 0,95 & 0,64 & 0,57 & 0,5 & 0,52 & 0,6 & 0,47 & 0,33 & 0,21 & 0,15 & 0,14 \\
\hline \multirow[t]{6}{*}{505 e J64 } & Minas Gerais & 1,22 & 0,09 & 0,73 & 0,6 & 1,28 & 4,55 & 6,58 & 5,7 & 5,24 & 1,67 & 1,66 \\
\hline & Maranhao & 1,41 & 0,35 & 0,68 & 0,32 & 0,31 & 5,57 & 7,95 & 2,82 & 2,5 & 1,1 & 0,27 \\
\hline & Goias & 2,93 & 1,08 & 1,77 & 3,22 & 1,25 & 31,6 & 8,41 & 2,83 & 3,04 & 2,72 & 2,4 \\
\hline & Rondonia & 0 & 0 & 1,25 & 1,29 & 5,05 & 19,89 & 58,74 & 23,23 & 11,93 & 13,87 & 1,05 \\
\hline & Santa Catarina & 3,18 & 0 & 2,16 & 1,18 & 2,9 & 16,58 & 16,92 & 13 & 8,69 & 5,29 & 2,98 \\
\hline & BRASIL & 0,89 & 0,94 & 0,51 & 0,73 & 0,92 & 6,87 & 6,54 & 4,63 & 3,94 & 2,25 & 1,14 \\
\hline \multirow[t]{5}{*}{ J65 } & Rio de Janeiro & $* * * * *$ & $* * * * *$ & $* * * * *$ & $* * * * *$ & $* * * * *$ & 0,1 & 0,1 & 0,09 & 0,18 & 0,27 & 0,09 \\
\hline & Sao Paulo & $* * * * *$ & $* * * * *$ & $* * * * *$ & $* * * * *$ & $* * * * *$ & 0,08 & 0,19 & 0,07 & 0 & 0,07 & 0,14 \\
\hline & Rio Grande do Sul & $* * * * *$ & $* * * * *$ & $* * * * *$ & $* * * * *$ & $* * * * *$ & 0,42 & 0 & 0 & 0,13 & 0,39 & 0,26 \\
\hline & Minas Gerais & $* * * * *$ & $* * * * *$ & $* * * * *$ & $* * * * *$ & $* * * * *$ & 0,51 & 0,08 & 0 & 0,08 & 0,38 & 0 \\
\hline & BRASIL & $* * * * *$ & $* * * * *$ & $* * * * *$ & $* * * * *$ & $* * * * *$ & 0,14 & 0,11 & 0,05 & 0,05 & 0,15 & 0,06 \\
\hline
\end{tabular}

Legenda: 500 e J60: Pneumoconiose dos Trabalhadores Expostos ao Carvão; 502 e J62.8: Pneumoconiose devido a outras sílicas e silicatos; 503 e J63.8: Pneumoconiose devido a outras poeiras inorgânicas; 505 e J64: Pneumoconiose não especificada e J65: Pneumoconiose associada a Tuberculose.

Caption: 500 and J60:Pneumoconiosis among Coal Workers; 502 and J62.8: Pneumoconiosis due to other silica or silicates; 503 and J63.8: Pneumoconiosis due to other inorganic dusts; 505 and J64: Unspecified pneumoconiosis and J65: Pneumoconiosis associated with tuberculosis.

Fonte: Calculadas com base na freqüência das internações registrada pelo DATASUS e a base populacional acima de 15 anos fornecidas e estimadas pelo IBGE. Source: calculations based on the frequency of hospitalizations recorded by DATASUS and population base above 15 years, supplied and estimated by IBGE.

Pneumoconiose associada à tuberculose (J65).

Verificou-se que os Estados de Santa Catarina e Rio Grande do Sul apresentaram maior freqüência de internações por Pneumoconiose dos Trabalhadores Expostos ao Carvão (códigos 500 e J60) no período compreendido entre 1993 e 1997, com queda a partir de 1998 .

Com relação à Silicose (502 e J62.8) notou-se baixas freqüências nos Estados do
Rio de Janeiro, Sergipe, Pernambuco e Rio Grande do Norte, mesmo apresentando registro de internações em todos os anos.

Surpreendentemente, o Estado de Minas Gerais, apesar de possuir o maior número de casos de silicose do país (segundo o Ministério da Saúde, até 1998 haviam sido diagnosticados mais de 7.416 casos de silicose na região de Nova Lima, área de mineração de ouro) ${ }^{8}$, houve apenas um registro de internação hospitalar no ano de 
2000, o que sugere a hipótese de ocorrência de equívocos na forma de coleta de dados primários do SUS ou de ausência de diagnóstico clínico.

O Estado de Mato Grosso apresentou maior coeficiente de Pneumoconiose derivada de outras poeiras inorgânicas (503 e J63.8), no período de 1993 a 2003, declinando a partir de 1999. Tanto a mineração quanto as indústrias da região podem ser responsáveis pelo adoecimento e conseqüentes internações por pneumoconiose na região ${ }^{5}$.

Os coeficientes de internações hospitalares por Pneumoconiose não especificada (505 e J64) indicaram altas freqüências em quase todos os Estados e anos estudados. Estes dados demonstram as possíveis dificuldades de diagnóstico específico das pneumoconioses. Tal fato explicaria, por exemplo, a ausência de dados para determinadas pneumoconioses e discrepâncias em outros resultados, assim como a possibilidade de repetição da mesma internação no ano. Tais variações poderiam se registrar em coeficientes de doenças agudas ou em casos de epidemias, e não em doenças de evolução lenta e crô- nica como as pneumoconioses.

Os dados sobre a Pneumoconiose associada à tuberculose (J65) mostraram tendência regular nas internações nos Estados do Rio de Janeiro e São Paulo, apesar dos baixos coeficientes.

A Tabela 2 demonstra os dados referentes a população economicamente ativa, número de trabalhadores formais e centros de referência em saúde do trabalhador distribuídos para o Brasil e suas macroregiões.

Observou-se que as Regiões Sul e Sudeste concentram um elevado número de trabalhadores formais por setor produtivo possuindo 88 dos 150 centros formalizados pelo SUS. Entretanto apesar da Região Centro-Oeste apresentar mais trabalhadores formais em relação a Região Norte, a quantidade de serviços em saúde do trabalhador não ultrapassa os 8 centros especializados.

O Gráfico 1 mostra a distribuição percentual por faixa etária em todo o período estudado, verificando-se que um pouco mais de $60 \%$ das internações por pneumoconiose ocorre em pessoas acima de 45 anos de idade, tendência esperada e

Tabela 2 - Número de população economicamente ativa, trabalhadores formais e centros de referência em saúde do trabalhador, Brasil e macro-regiões.

Table 2 - Number of economically active people, formal workers and reference healthcare centers for workers, Brazil and macro-regions.

\begin{tabular}{|c|c|c|c|c|c|c|c|}
\hline & \multirow[t]{2}{*}{ PEA $^{1}$} & \multicolumn{5}{|c|}{ Indústria - Setor produtivo ${ }^{2}$} & \multirow[t]{2}{*}{ CEREST $^{3}$} \\
\hline & & Ind. Ext. Min. & Ind. Transf. & Serv. Ind. UP & Constr. Civil & TOTAL & \\
\hline NORTE & 6.499 .000 & 6.487 & 181.615 & 15.621 & 47.785 & 251.508 & 13 \\
\hline NORDESTE & 23.898 .000 & 21.695 & 659.487 & 63.467 & 195.067 & 939.746 & 41 \\
\hline CENTRO-OESTE & 6.341 .000 & 6.914 & 244.328 & 23.542 & 83.124 & 357.908 & 8 \\
\hline SUDESTE & 38.813 .000 & 73.526 & 2.838 .592 & 162.966 & 556.372 & 3.631 .456 & 71 \\
\hline SUL & 14.607 .000 & 14.184 & 1.432 .137 & 53.472 & 165.873 & 1.665 .666 & 17 \\
\hline BRASIL & 90.158 .000 & 122.806 & 5.356 .159 & 319.068 & 1.048 .251 & 6.846 .284 & 150 \\
\hline
\end{tabular}

Legenda: Ind. Ext. Min: Indústria Extrativa Mineral; Ind. Transf.: Indústria da Transformação; Serv. Ind. UP: Serviços Industriais de Utilidade Pública; Constr. Civil: Construção Civil.

Fontes: IBGE, RAIS - Ministério do Trabalho e Emprego - MTE e FormSUS - Ministério da Saúde - MS

Notas: ${ }^{1}$ População Economicamente Ativa estimada em 2003

2 Número de trabalhadores formais em 2003

${ }^{3}$ Centros de Referência em Saúde do Trabalhador formalizados no SUS em 2006

Caption: Ind. Ext. Min: Mineral Extractive Industry; Ind. Transf.: Transformation Industry; Serv. Ind. UP: Public Utility Industrial Services; Constr. Civil: Civil Construction.

Source: IBGE, RAIS - Work Ministry and FormSUS - Ministry of Health

Notes: 'estimated economically active population in 2003

${ }^{2}$ Number of formal workers in 2003

${ }^{3}$ Centers of Reference in Health of the Worker legalized in the SUS in 2006 


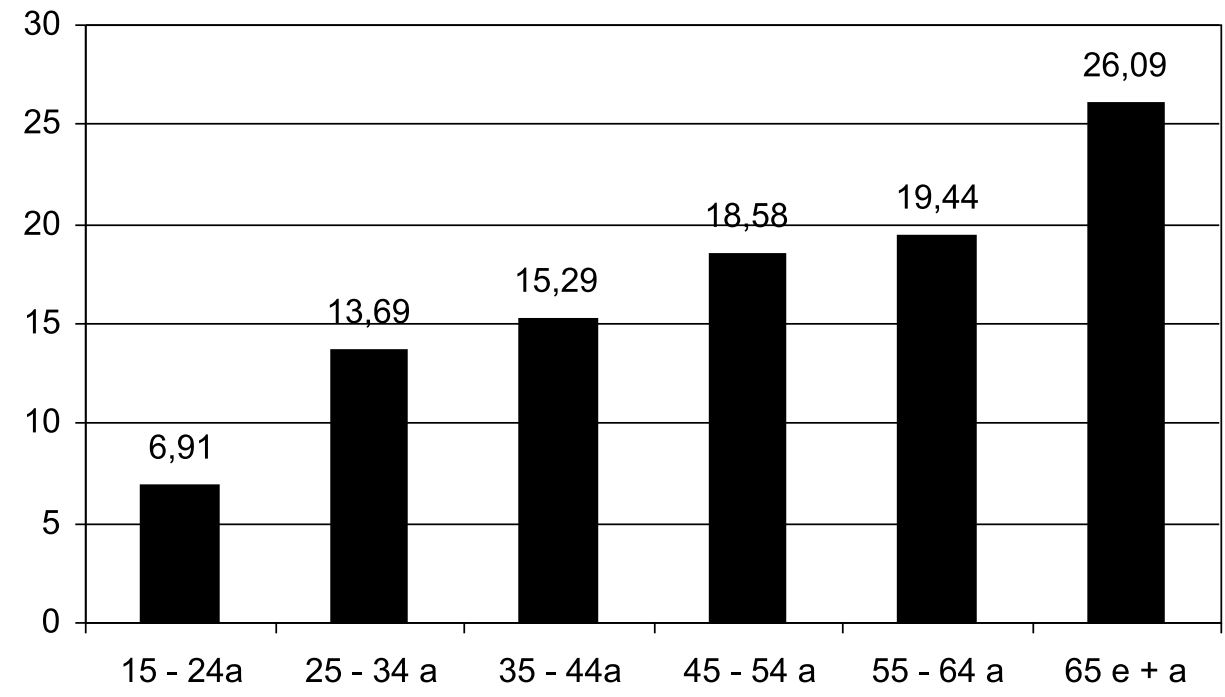

Gráfico 1 - Distribuição percentual por faixa etária das internações hospitalares por pneumoconioses no Brasil, período entre 1993 a 2003.

Chart 1 - Percentage distribution of hospital admissions due to pneumoconiosis by age group in Brazil, for the period between 1993 and 2003.

que pode ser explicada pelo longo período de latência da doença e pelo próprio processo de adoecimento, em geral mais grave nos idosos.

A distribuição por sexo (Gráfico 2), mostra que $63 \%$ das internações são de homens e $37 \%$ de mulheres. A concentração de mãode-obra do sexo masculino nas atividades com risco de exposição, como ocorre na mineração e na construção civil, concorda com a distribuição encontrada. Entretanto, é também freqüente a mão-de-obra feminina em atividades de risco, como a indústria têxtil de amianto.

\section{Discussão}

Uma análise preliminar do Mapa 1 permitiu identificar que os dados refletem os efeitos do processo industrial e extrativista, ambos presentes nos Estados da Região Sudeste, que é a mais populosa das Regiões e com o maior número de empregados formais. Segundo o IBGE, possui uma rede de assistência diferenciada com diversos Estados possuindo programas de Saúde do Trabalhador como Minas Gerais, Rio de Janeiro e São Paulo 5 .

O sul caracteriza-se por ser uma região

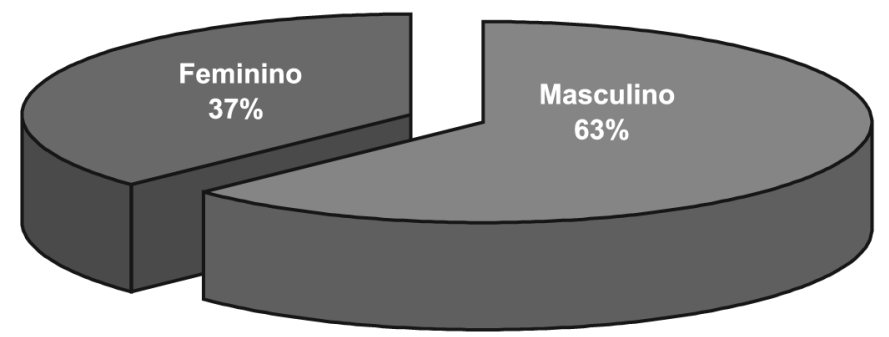

Gráfico 2 - Distribuição percentual por sexo das internações hospitalares por pneumoconioses no Brasil, período entre 1993 e 2003.

Chart 2 - Percentage distribution of hospital admissions due to pneumoconiosis by gender in Brazil, period between 1993 and 2003. 
com mineração de carvão e grande número de indústrias com exposição a poeiras causadoras de pneumoconiose. Em 1991, Souza Filho e cols. estudaram 92 casos de fibrose maciça pulmonar progressiva, correspondentes a $6 \%$ de 1.500 casos de pneumoconiose dos trabalhadores das minas de carvão, fluorita, cerâmica, jato de areia e moagem de pedra da região carbonífera do sul do Estado de Santa Catarina, em um período de 14 anos de observação ${ }^{6}$. Algranti e cols. estimaram uma prevalência pontual para pneumoconiose do trabalhador de carvão de $5,6 \%$ de uma amostra original de 956 mineiros ${ }^{7}$.

O baixo índice de notificação das internações na Região Norte e em alguns Estados do Nordeste ocorre possivelmente devido à falta de informação, acesso dos trabalhadores aos serviços de saúde, dificuldade de diagnóstico e redução dos leitos hospitalares na região. Sabe-se que os Estados do Pará e Amazonas possuem programas de Saúde do Trabalhador, e na década de 90 apresentaram um aumento nos índices de internação indicando uma possível melhora na rede de atenção na região ${ }^{5}$. Denota-se a importância da presença de serviços específicos em doenças ocupacionais como os Centros de Referências em Saúde do Trabalhador (CEREST). Na Tabela 2 foi possível perceber a dificuldade de acesso dos trabalhadores aos serviços de saúde especializados. Em Regiões como Norte e Centro-Oeste, com um número elevado de trabalhadores formais com risco de desenvolver tais doenças ,verificou-se que a quantidade de centros de referência não abrange a demanda disponível.

A ocorrência de significativa freqüência de internações por Pneumoconiose dos Trabalhadores Expostos ao Carvão nos Estados de Santa Catarina e Rio Grande do Sul pode ser explicada pela presença de mineração de carvão e de um grande número de indústrias onde ocorre a exposição às poeiras causadoras de pneumoconioses. A queda a partir do ano de 1998 relaciona-se, provavelmente, às mudanças de critérios diagnósticos ou à forma de coleta dos dados primários pelo SUS, dificultada pela transição para a CID 10, que sofreu significativa modificação na estrutura de codificação.

O Nordeste vivenciou no início dos anos 90 um aumento de casos de silicose relacionado à atividade de cavar poços. No Ceará, região de Tianguá, Holanda e cols. realizaram avaliação pneumológica em 360 cavadores de poços, dos quais 63 apresentaram radiografias de tórax compatíveis com silicose. Em 1999, esses mesmos autores mostraram os casos diagnosticados no período de 1986 a 1989, entre 687 daqueles profissionais. A ocorrência de silicose foi de $26,4 \%$ (180 casos) com uma prevalência de silicotuberculose de $7,2 \%$ entre os mesmos ${ }^{4}$.

No Estado do Rio de Janeiro, a atividade de jateamento de areia foi a responsável por muitos casos de silicose no início dos anos $90^{5}$. A primeira publicação sobre casos de silicose entre jateadores de areia foi em 1984, quando Marchiori e cols. relataram 4 casos de silicose ${ }^{10}$. Especificamente na Indústria Naval, em 1995, foram encontrados 138 casos $(23,6 \%)$ entre 586 radiografias de trabalhadores expostos ao jato de areia $^{11}$. Em um serviço de referência ambulatorial para pneumoconioses foram avaliados 457 trabalhadores expostos à sílica, e diagnosticados 104 casos de silicose entre 1992 e 2002, oriundos, principalmente, dos estaleiros do Estado do Rio de Janeiro ${ }^{12}$. Ferreira e cols. realizaram recentemente um estudo de subpopulações de linfócitos no lavado brônquico de 26 casos de asbestose, entre 58 trabalhadores expostos na indústria têxtil de amianto ${ }^{13}$.

Em São Paulo, no ano de 1980, um estudo sobre a fisiopatologia da silicose mostrou 101 casos entre trabalhadores ceramistas $^{14}$. Outro estudo entre ceramistas foi publicado em 1981 por Nogueira e cols. quando, a partir da descoberta de um caso, foram encontrados diversos focos de silicose nas indústrias de cerâmica no Es$\operatorname{tado}^{15}$. Entretanto, as freqüências de internações referentes ao Estado de São 
Paulo foram baixas e não foram registradas na Tabela 1.

Recente levantamento realizado no ambulatório do Hospital das Clínicas da UFMG, entre os trabalhadores atendidos no período entre 1989 e 2000, foram diagnosticados 126 casos de silicose, sendo a mineração a principal atividade desses trabalhadores, seguidos por trabalhadores de indústria da cerâmica, lapidação e metalurgia ${ }^{9}$. Possivelmente, os casos de silicose, no Estado de Minas Gerais, podem ter sido codificados como pneumoconiose não especificada, onde, de acordo com a Tabela 1 , mostra taxas entre 6,58 no ano 2000 e 4,55 em 1998, explicando o único registro encontrado no ano de 2000.

Com relação à Pneumoconiose associada à Tuberculose, é conveniente pensar em subregistro, pois, de acordo com o Ministério da Saúde (2000), a incidência média de Tuberculose na Região Sudeste é de 55:100.000 habitantes/ano. E, embora a maior incidência e prevalência da tuberculose estejam no Estado do Rio de Janeiro, São Paulo concentra o maior número de indústrias consideradas de risco para as pneumoconioses, reconhecido pelo seu desenvolvimento industrial e apresentando em seu território indústrias automobilísticas, da cerâmica, têxteis, entre outras ${ }^{5}$. Assim, a associação destas duas doenças deve ser freqüente em ambos os Estados, sendo, porém, pouco diagnosticada.

Apesar de a literatura brasileira apontar diversos casos de asbestose como os descritos no Estado de São Paulo, em 2001, onde foram diagnosticados 74 (8,9\%) casos em uma população de 828 trabalhadores expostos ao amianto ${ }^{16}$, e no Estado do Rio de Janeiro, encontraram 26 (44,8\%) casos entre 58 trabalhadores expostos ${ }^{17}$, os mesmos não aparecem registrados no $\mathrm{SIH} /$ SUS, podendo refletir uma dificuldade de diagnóstico ou falta de acesso desses tra- balhadores aos serviços de saúde. Outra hipótese seria a codificação desses casos como pneumoconiose não especificada.

\section{Conclusão}

A distribuição das internações por pneumoconioses no Brasil é heterogênea e possivelmente reflete o vértice de uma pirâmide invertida do total de casos que incidem entre os trabalhadores brasileiros. Primeiro, as internações representam casos graves ambulatoriais em estágios mais avançados de evolução e com complicações cardiopulmonares, não expressando a freqüência real de doentes. Além disso, o acesso aos serviços de saúde ainda não garante a efetividade necessária no diagnóstico e registro de casos. Erros na codificação e dificuldades de diagnóstico podem ainda mascarar a totalidade dos casos de internações.

Esse estudo pretendeu contribuir para a construção de um panorama epidemiológico das pneumoconioses a partir da freqüência com que os trabalhadores doentes se internam na rede pública de assistência hospitalar. Entretanto, confrontando com a experiência dos pesquisadores, permitiu inferir que há baixa sensibilidade no SUS para identificar os casos, possivelmente diagnosticados como pneumopatias, com as quais os profissionais estão mais familiarizados.

Assim, reitera-se que as pneumoconioses são um importante problema de Saúde Pública, ainda não refletido adequadamente pelos números registrados. Seu enfrentamento, ao lado de propiciar uma aproximação da real situação epidemiológica, contribuirá para orientar as medidas profiláticas de redução dos riscos e preparar o sistema assistencial para melhor atender aos doentes. 


\section{Referências}

1. Mossman, BT, Churg, A - Mechanism in the pathogenesis of asbestosis and silicosis. Am J Resp Crit Care Med 1998; 157(5): 1666-80.

2. Algranti, E. Epidemiologia das doenças ocupacionais respiratórias no Brasil. Epidemiologia das doenças respiratórias 2001; 1(3): 119-43.

3. Giannasi, F. Occupational Exposures to Asbestos in Brazil. Int J Occup Environ Health 1997; 3(2): 150-7.

4. Holanda, MA; Martins, MPS; Felismino, PH; Pinheiro, VG. Silicose em cavadores de poços da região de Ibiapaba (CE): da descoberta ao controle. J Pneumol 1999; 25(1): 111.

5. Castro, HA; Vicentin, G; Silva, CG.. Estudo das internações hospitalares por Pneumoconioses no Brasil período 1984/2003. Rev Bras Epidemiol 2005; 8(2): xxx.

6. Souza Filho AJ, Alice SH. Fibrose maciça pulmonar progressiva. J Pneumol 1991; 17(4): 147-53.

7. Algranti E, Souza Filho AJ, Mendonça EMC, Silva RCC, Alice SH. Pneumoconiose de mineiros de carvão: dados epidemiológicos de minas da bacia carbonífera brasileira. J Pneumol 1995: 21(1): 9-12.

8. Carneiro APS, Campos LO, Gomes MFCF, Assunção AA. Perfil de 300 trabalhadores expostos à sílica atendidos ambulatorialmente em Belo Horizonte. J Pneumol 2002; 28(6): 329-34.

9. Algranti E, Freitas JBP, Mendonça, EMC, De Capitani EM, Silva HC, Bussacos MA. Non-malignant asbestos-related diseases in Brazilian asbestos-cement workers. Am J Ind Med 2001; 40: 240-54.
10. Marchiori E, De Luca V. Silicose aguda em ajatadores de areia (Considerações sobre quatro casos). J Pneumol 1984: 9(3): 139-44.

11. Castro HÁ, Bethlem EP. A silicose na indústria naval do Estado do Rio de Janeiro: Comissão técnica Estadual de Pneumopatias Ocupacionais do Estado do Rio de Janeiro, análise parcial. J Pneumol 1995; 21: 13-6.

12. Castro HA, Vicentin G, Ribeiro PC, Mendonça ICT. Perfil respiratório de 457 trabalhadores expostos à poeira de sílica livre no Estado do Rio de Janeiro. Pulmão RJ 2004; 13(2): 81-5.

13. Ferreira A, Moreira JS, Caetano R, Gabetto JM, QuiricoSantos T. Caracterização imunofenotípica das subpopulações de linfócitos do lavado broncoalveolar de pacientes com silicose. J Pneumol 2000; 26(3): 107-12.

14. Ribeiro HP, Koga RK, Dos Santos R. Fisiopatologia da silicose em ceramistas. Estudo de 101 casos. J Pneumol 1980; 6(3): 121-8.

15. Nogueira DP, Certain D, Brolio R, Garrafa NM, Shibata H. Ocorrência de silicose entre trabalhadores da indústria cerâmica da cidade de Jundiaí, SP (Brasil). Rev Saúde Pública 1981; 15(3): 263-71.

16. MS/FNS, Manual de Normas para o Controle das Pneumoconioses. Brasília; 1997.

17. Mogami R, Marchiori E, Albuquerque HA, Ribeiro $P$, Capone D. Correlação entre radiografia convencional e tomografia computadorizada de tórax em trabalhadores da indústria têxtil do asbesto. Rev Imagem 2001; 23 (4): 233-8

Recebido em: 08/01/07 Versão final reapresentada em: 04/06/07 Aprovado em: 08/06/07 